\title{
The Myosin II Coiled-Coil Domain Atomic Structure in its Native Environment
}

Hamidreza Rahmani ${ }^{1}$, Wen $\mathrm{Ma}^{2}$, Zhongjun $\mathrm{Hu}^{3}$, Nadia Daneshparvar ${ }^{1}$, Dianne Taylor ${ }^{4}$, J. Andrew McCammon $^{5}$, Thomas Irving ${ }^{6}$, Robert Edwards ${ }^{7}$ and Kenneth Taylor ${ }^{1}$

${ }^{1}$ Florida State University, United States, ${ }^{2}$ University of California San Diego, United States, ${ }^{3}$ Facebook, United States, ${ }^{4}$ florida state university, United States, ${ }^{5}$ University of California, San Diego, United States, ${ }^{6}$ Illinois Institute of Technology, United States, ${ }^{7}$ Duke University, United States

The first atomic model for the myosin coiled-coil in its native environment is reported(1). The coiled-coil structure as a packing arrangement for $\alpha$-helices was predicted by Francis Crick (2) to explain the X-ray diffraction patterns of fibrous proteins first observed by Astbury (3). Later the same pattern was observed in globular proteins like myoglobin and hemoglobin making this specific kind of $\alpha$-helix packing a focus

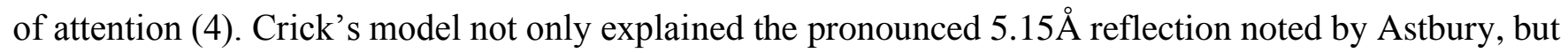
also laid out the heptad pattern for the coiled-coil sequence, remarkably decades before any coiled coil proteins were sequenced! Noticing the fraction of hydrophobic amino acids in tropomyosin Crick predicted the existence of a heptad pattern in the (2). Parallel, two-stranded coiled-coils, such as those of myosin and tropomyosin, have a distinct sequence pattern: a heptad repeat with hydrophobic residues in the first and fourth position. Hydrophobic residues following the heptad pattern are positioned at the interface of the two 7/2 $\alpha$-helices, which Crick described as having "knobs-in-holes" packing. Interlocking residues in this manner plays a significant part in stabilizing $\alpha$-helices in a large variety of proteins (5).

Coiled-coil proteins come in a remarkable variety of lengths from tens of residues to more than one thousand in proteins such as myosin II. NuMA and Giantin are the only proteins with coiled coil domains longer than myosin's (6) but our analysis using PairCoil (7) indicates their coiled-coil region might be interrupted by uncoiled sub-regions making the myosin II coiled coil possibly the longest uninterrupted coiled coil in nature. For that exact reason, this molecule is too large to be resolved in one piece under an electron microscope with sufficient magnification. The helical symmetry in insect, asynchronous flight muscle myosin filaments provides a unique opportunity to resolve a long coiled-coil in one piece by reconstructing a smaller segment and expanding the map by imposing helical symmetry (Fig. 1B).

Unlike actin filaments, which across muscle types are very similar, myosin filaments show a high degree of structural diversity. Differences between muscle characteristics (beat frequency in insect flight muscle, produced force, etc.) is likely to come from myosin filaments which are less known in the backbone region. There are close to a thousand disease-causing mutation-sites in the human beta-cardiac myosin and about half of the are in the coiled-coil region (8). The tail region of the straited muscle myosin is highly conserved and all changes are point mutations, not insertions, deletions or early termination. The conservation is higher among the charged residues, even higher than hydrophobic residues that keep the coiled coil together. This is because the myosin tails form the filament through charged interactions.

We collected a larger data set where 2993 micrographs with filaments were acquired from the grid using a DE64 direct electron detector. The collection was done in integration mode using leginon/appion 
pipeline software, with the dose of $60 \mathrm{e}-/ \AA 22$. Filaments were picked manually andcut into 216,045 helical segments with one crown (145 $\AA$ axial repeat) unit per segment. The helical segments were using relion (9) to reserve the prior information of the segments. cisTEM was used to perform 2D classification (10) and the classes with pronounced head appearance were selected for the final stack. The final refinement was done on 173,515 segments and yielded a $4.25 \AA$ structure. Helical parameters were calculated using relion_helix_toolbox (11) and the helical rise was $149.56 \AA$ and the helical twist was $34.08^{\circ}$. The structure was reconstructed under $\mathrm{C} 4$ rotational symmetry since cisTEM does not perform iterative helical realspace reconstruction. Sharpening was done using local-Deblur and embfactor and both produced similar results, but local-Deblur was more effective in keeping the $\mathrm{S} 2$ region and resolving the large side chains. Atomic model was built de novo with no constraints and refined on Phenix (Fig. 1C). Our atomic model was validated using CCCP server (12).

Presence of a two-stranded $\alpha$-helical coiled coil is observed with a $5.15 \AA$ near meridional reflection in the $\mathrm{X}$-ray fiber diagram (13). Wide angle $\mathrm{X}$-ray fiber diagrams of Lethocerus indicus flight muscle reveal the distinctive 5.15 ̊ layer line (Fig. 1A). After helically extending and projecting the reconstruction (Fig. 1B), the resulting power spectrum also reveals the characteristic $5.15 \AA$ peak of the coiled coil and equatorial or near-equatorial intensities at $10 \AA$ (Fig. 1C).

Building and validating the atomic model showed a high degree of similarity between our atomic model and crystal structures (14) of human cardiac myosin tail segments, except in Skips 2 and 4 (Fig 1 D,E). In Skip 2 our atomic model shows a canonical coiled coil instead of two parallel $\alpha$-helices and in Skip 4, we have one unfolded chain unlike the crystal structure where both chains are unfolded. MD simulations showed that the model becomes more similar to the crystal structure in solution. In these areas the structure deviates from what is expected based on the sequence because of the interactions with other proteins and myosin tails.

Cricks parametrization server also reports the rise-per-residue values and the numbers for atomic models showing possible places that the coiled coil length could change. This change is enough to provide the 143 to $145 \AA$ shift that is observed X-ray fiber diffraction of contracting muscle (Fig. 2). The diffraction data is consistent with a local change rather than a uniform stretching along the entire coiled coil. In Skips 2 and 4 the difference between the atomic model, crystal structures, and the MD simulation results could explain what is observed in the contraction cycle. 


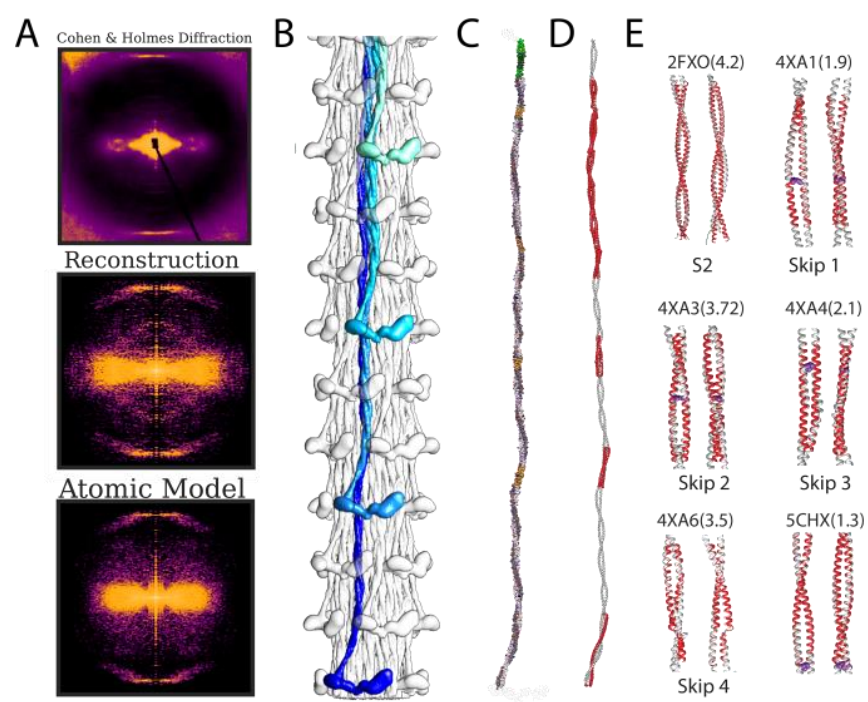

Figure 1. (A) Muscle X-ray fiber diffraction pattern [13] compared to the power spectrum of our reconstruction and the atomic model. (B) Extended map and the segmentation used to extract a myosin tail. (C) the atomic model as a very long coiled coil. (D) Overlap of crystallized segments of the human myosin on our atomic model. (E) RMSD, in parentheses, between important crystallized segments and our model.

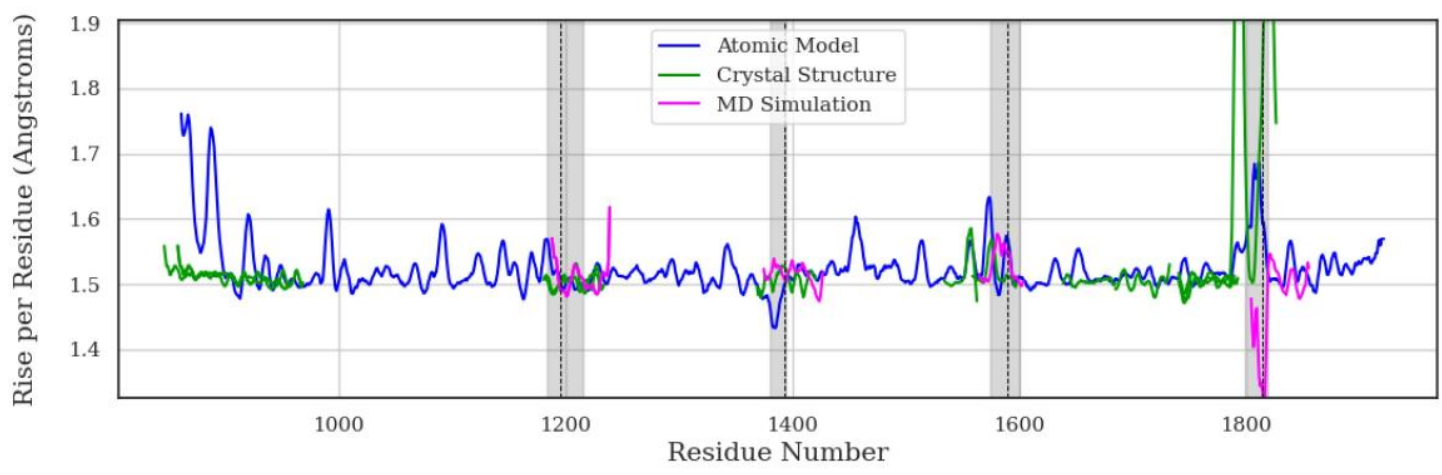

Figure 2. Rise-per-residue calculated for our atomic model, crystal structures, and MD simulation results. Skip 2 is the only region showing a significant decrease in the rise-per-residue and Skip 4 shows the most change before and after the MD simulation.

\section{References}

1. W. M. Hamidreza Rahmani, Zhongjun Hu, Nadia Daneshparvar, Dianne W. Taylor, J. Andrew McCammon, Thomas C. Irving, Robert J. Edwards and Kenneth A. Taylor, The Myosin II Coiled-Coil Domain Atomic Structure in its Native Environment. PNAS 118, no. 14 (2021).

2. F. H. C. Crick, The packing of a-helices: Simple coiled coils. Acta Crystallogr 6, 689-697 (1953). 3. W. T. Astbury, On the structure of biological fibres and the problem of muscle. Proc Royal Soc London. B 134, 303-328 (1947).

4. M. Gruber, A. N. Lupas, Historical review: another 50th anniversary-new periodicities in coiled coils. Trends in biochemical sciences 28, 679-685 (2003).

5. A. Lupas, Coiled coils: new structures and new functions. Trends Biochem Sci 21, 375-382 (1996). 
6. L. Truebestein, T. A. Leonard, Coiled-coils: The long and short of it. Bioessays 38, 903-916 (2016).

7. A. V. McDonnell, T. Jiang, A. E. Keating, B. Berger, Paircoil2: improved prediction of coiled coils from sequence. Bioinformatics 22, 356-358 (2006).

8. F. Parker, M. Peckham, Disease mutations in striated muscle myosins. Biophys Rev, 1-8 (2020).

9. S. H. Scheres, A Bayesian view on cryo-EM structure determination. Journal of molecular biology 415, 406-418 (2012).

10. T. Grant, A. Rohou, N. Grigorieff, cisTEM, user-friendly software for single-particle image processing. Elife 7 (2018).

11. S. He, S. H. Scheres, Helical reconstruction in RELION. J. Struct. Biol. 198, 163-176 (2017).

12. N. W. Schmidt, G. Grigoryan, W. F. DeGrado, The accommodation index measures the perturbation associated with insertions and deletions in coiled-coils: Application to understand signaling in histidine kinases. Protein Sci 26, 414-435 (2017).

13. C. Cohen, K. C. Holmes, X-ray diffraction evidence for alpha-helical coiled-coils in native muscle. J Mol Biol 6, 423-432 (1963).

14. K. C. Taylor et al., Skip residues modulate the structural properties of the myosin rod and guide thick filament assembly. Proc Natl Acad Sci U S A 112, E3806-3815 (2015). 